Rapid Reviews COVID-19

\title{
Review 1: "Integrated \\ Single-Cell Atlases Reveal \\ an Oral SARS-CoV-2 \\ Infection and Transmission \\ Axis"
}

\section{Hongxiang Liu ${ }^{1}$, Zhonghou Wang, Wenxin Yu, Mohamed Ishan}

${ }^{1}$ Associate Professor, University of Georgia, Regenerative Bioscience Center, Animal and Dairy Science

Published on: Jan 18, 2021

DOI: $10.1162 / 2 \mathrm{e} 3983 f 5 . d e 4722 \mathrm{df}$

License: Creative Commons Attribution 4.0 International License(CC-BY 4.0) 


\section{$\underline{\text { RR:C19 Evidence Scale rating by reviewer: }}$}

- Reliable. The main study claims are generally justified by its methods and data. The results and conclusions are likely to be similar to the hypothetical ideal study. There are some minor caveats or limitations, but they would/do not change the major claims of the study. The study provides sufficient strength of evidence on its own that its main claims should be considered actionable, with some room for future revision.

$* * * * * * * * * * * * * * * * * * * * * * * * * * * * * * * * * * * * * * *$

\section{Review:}

In this manuscript entitled "Integrated Single-Cell Atlases Reveal an Oral SARS-CoV-2 Infection and Transmission Axis", the authors "Huang N, Perez P, Kato T, Mikami Y et al" aimed to address that the oral cavity represents a robust site for COVID-19 infection and implicates saliva in viral transmission.

Evidence is reliable. The claims are generally supported by the data and analytic methods used. Decision-makers should consider the claims in this study as actionable after revisions.

The study analyzed more sites of tissues in the oral cavity than previous report to demonstrate oral cavity represents a route for COVID-19 infection. What makes the current study strong is the finding of viruses in the in situ oral epithelial cells and shed cells in saliva, which implicates saliva in viral transmission. The article is well structured. However, revisions are needed before publication. Concerns/suggestions are as below:

(1) In figure 1a, a possibility that viruses enter blood stream needs to be considered and added;

(2) In multiple figures, the grey colored signals in the following images are difficult to recognize (Fig 5a: Ace2; Fig 5d: Spike; Supp Fig 4c-d: Ace2; Supp Fig 5a: ACE2; Supp Fig 5d: TMPRSS4; Supp Fig 6b: ACE2; Supp Fig 6e: SARS-CoV-2). The authors may consider trying blue or another color for these signals, and use grey color for DAPI signals in nuclei.

(3) In Figure 3d-e and Supp. Figure 3 (dorsal tongue data), annotation of some cell clusters are not very accurate or lack solid evidence, e.g. fungiform diff1 and 
fungiform diff2. If annotations are speculated from literature, authors need to cite the appropriate publications and briefly describe how to annotate the cells.

(4) In Fig 5b and Supp Fig 5b, normal control tissue is needed for comparison and supporting claims "Inflammatory responses to the presence of virus in the glands ranged from mild to severe in SGs (Fig 5b; Supp Fig 5b).”

(5) Supp Fig 5h (mentioned in legend) and Fig 6h (mentioned in results) are missing;

(6) In Supp Fig 6, scale bar (a,e) is likely meant to be (b,e);

(7) To be reader-friendly, it will be great to have a list of abbreviations if the journal allows;

(8) The term "saliva glands" needs to be corrected to "salivary glands"; minor and major SG need to be clarified in the text of the entire article;

(9) In Discussion, page 9, left column, the last sentence is difficult to understand and does not seem to be reasonable;

(10) Many citations don't have volume, issue and page numbers.

(11) In Result, page 7, left column, first paragraph, the claim of last sentence is true for TMPRSS, but not ACE2 (Fig 4d). ACE2 is robust in both SB and Basal.

(12) In Result, page 7, left column, second paragraph, second sentence, the claim is not supported by the data shown in Fig 4 e.

(13) Overall, clarity needs to be improved; typing and grammatical errors are to be corrected. 